\title{
Distinct new catalysts for utilization of ethylene gas to polymer and oligomer
}

\author{
Abdulhamid A. Alsaygh
}

Received: 25 June 2013/Accepted: 24 September 2013/Published online: 9 October 2013

(c) The Author(s) 2013. This article is published with open access at Springerlink.com

\begin{abstract}
Bi-nickel-center catalysts were prepared by Schiff-base condensation of 2,3-butanedione with 2,6-diisopropylaniline; 2-5-butylaniline, 2-isopropylaniline and 1,4-phenylene diamine(I). More Schiff-base ligands were prepared by condensation of 2,3-butandione with 2,6-diisopropylaniline; 2,4-6-trimethyl aniline and 4,4-diaminophenyl (benzidine)(II), and subsequent metathesis reaction with (DME)NiBr2. Bis(alpha diimine)nickel(II)dibromide complexes are suitable catalysts and are precursors for the polymerization of ethylene after activation with methylaluminoxane (MAO). In comparison with these complex analogs, mono-nickel-center catalysts, the new catalysts have much bigger molecules, illustrated by the distance between every two active centers (II and III). For bi-nickel-center catalysts, the catalyst's structural credibility had profound influence on the catalytic activity. When the substituent was diisopropyl; $t$-butyl or methyl, the catalysts demonstrated much higher catalytic activity than the corresponding mononickel-center catalysts. Catalyst (II) with phenyl bridge showed high activity compared with catalyst (III) with diphenyl bridge. The catalytic properties of these complexes and the character of the obtained polymers depend on the ligand structure of the used catalysts. Substituents on the arene moiety and/or the backbone of the ligand influence the polymerization reaction. Small aryl substituents result in the formation of low molecular weight oligomers, whereas bulky aryl substituents gave high molecular weight polyethylene. Catalysts are cheap and can be prepared easily with available starting material and stable in air. The effect of $\mathrm{Al} /$
\end{abstract}

\footnotetext{
A. A. Alsaygh $(\bowtie)$

Petrochemical Institute at King Abdulaziz City for and Technology, PO Box 6086, Riyadh 11442, Saudi Arabia e-mail: aalsaygh@kacst.edu.sa
}

$\mathrm{Ni}$ ratio, of reaction time, variation of ethylene pressure, and the effect of temperature on catalyst performance will be discussed.

Keywords Organometallic - Polymerizations · Late transition metals · Ethylene

\section{Introduction}

The area of olefin polymerization catalysis has endured a remarkable increase in research outcome over the past 20 years, with many academic and industrial research centers involving in the design of a new organometallic precatalysts for the controlled synthesis of polyolefin products. In the early 1970 s researches on the Group 4 metallocenes gave more detailed understanding of the chemistry around of the activated sites [6]. In the early 1990s new classes of "post-metallocene" catalysts were developed which overcame the inherent problems in Group 4 cyclopentadienyl systems, by using late transition metal systems enabling the incorporation of polar comonomers into polyolefin materials, and gave a new morphology surface at low levels of comonomer incorporation and changed the bulk properties of the polyolefin at higher levels of incorporation [2, 5]. In the late 1990s, a new type of catalysts was developed, based on late transition complexes such as nickels' which are very active towards ethylene polymerizations coupled with, the last decade, breakthrough by utilizing such a system to do what is so called living polymerization of olefinic monomers. In this process, the absence of chain-transfer or chain-termination gives rise to a polyolefinic material with very narrow molecular weight distributions (typically $<1.1$ ), block copolymers, and polymers with novel topologies $[3,4,7]$. 

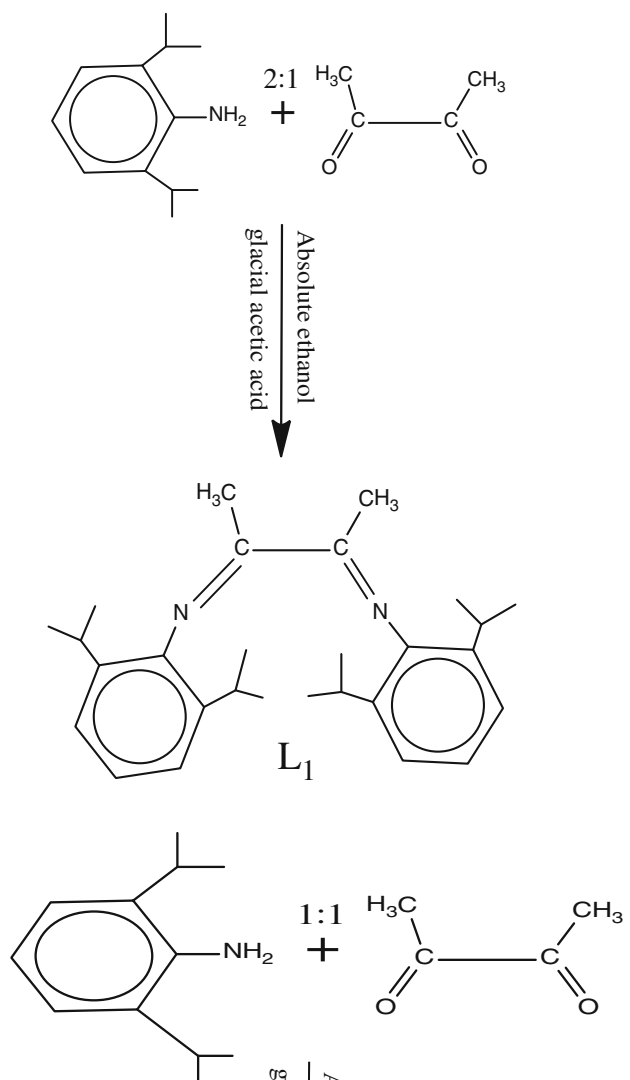

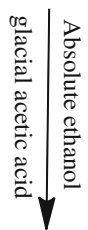

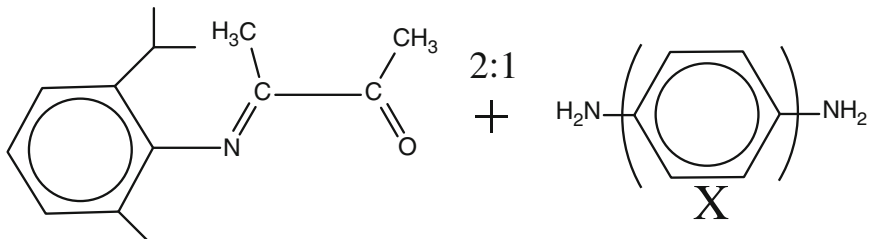

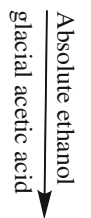

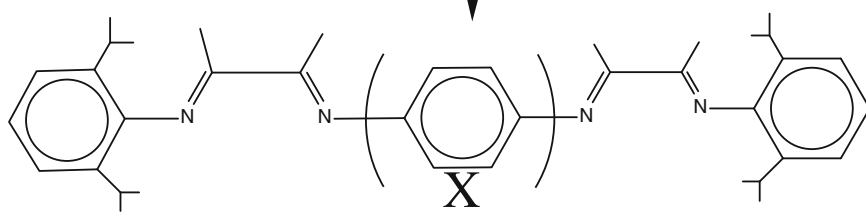

$$
\begin{aligned}
& X=@ \mathrm{~L}_{2} \\
& \mathrm{X}=\square \mathrm{L}_{3}
\end{aligned}
$$

Scheme 1 Synthesis of ligands $\mathbf{L}_{\mathbf{1}}, \mathbf{L}_{\mathbf{2}}$ and $\mathbf{L}_{\mathbf{3}}$ 


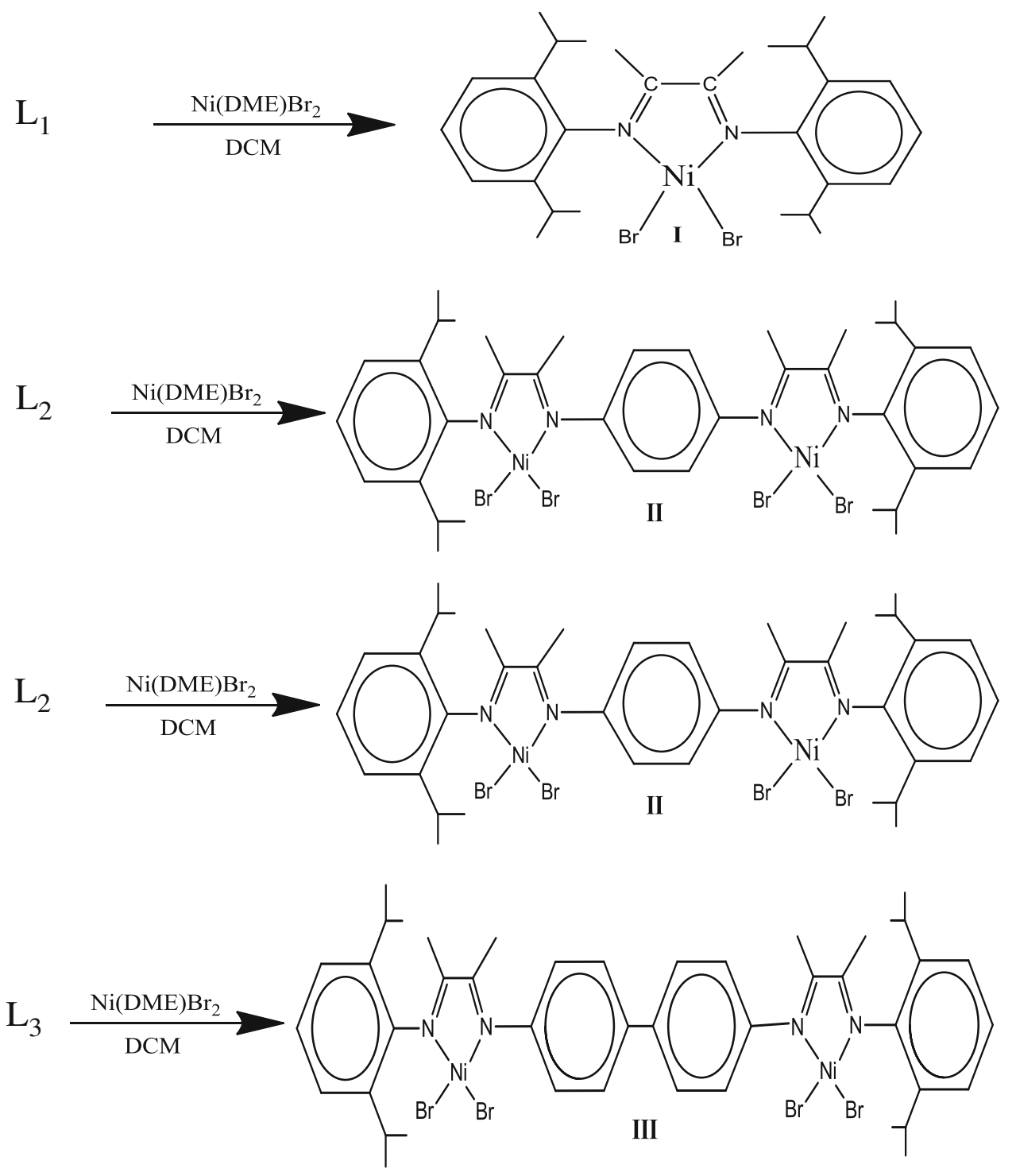

Scheme 2 Synthesis nickel complexes 1, 2, and $\mathbf{3}$

\section{Experimental}

All manipulations of air and/or water-sensitive compounds were carried out under dry nitrogen atmosphere using a dry box or Schlenk line techniques. The molecular weight (MW) and polydispersity index (PDI) of PE were determined by GPC (PL-GPC220/FTIR) in 1,2,4-trichlorobenzene/THF using polystyrene columns as standard.

Synthesis of new ligands

The ligand $\mathbf{L}_{\mathbf{1}}$ was synthesized by condensation of 2,3butanedione with 2,6-di-isopropylaniline; 2-5-t-butylaniline, 2-isopropylaniline and 1,4-phenylene diamine. More Schiff-base ligands, $\mathbf{L}_{\mathbf{2}}$ and $\mathbf{L}_{\mathbf{3}}$, were prepared by condensation of 2,3-butandione with 2,6-diisopropylaniline; 2,4-6-trimethyl aniline and 4,4-diaminophenyl (benzidine)(II), (Scheme 1). For example, a 100-ml Schlenk flask was charged with $3.29 \mathrm{~g}(18.59 \mathrm{mmol})$ (2,6-di-isopropylaniline; 2-5-t-butylaniline, 2-isopropylanilin, or 2,46-trimethyl aniline), $1.60 \mathrm{~g}$ (18.59 $\mathrm{mmol})$ 2,3-butanedione, $30 \mathrm{ml}$ ethanol and $0.4 \mathrm{ml}$ glacial acetic acid, the pale yellow solution was stirred for one day at room temperature (the intermediate products were not isolated). Then, $9.30 \mathrm{mmol}$ of 4,4-diaminophenyl or benzidine, and more $30 \mathrm{ml}$ of ethanol was added. The final solution was stirred for 1 week at room temperature which yielded an yellow precipitate. The yellow precipitate was isolated by filtration, washed with $2 \times 10 \mathrm{ml}$ ethanol, and dried in vacuum; $4.8 \mathrm{~g}$ yellow powder was obtained; yield, $63 \%$. 
Synthesis of complexes $\mathbf{1}, \mathbf{2}$ and $\mathbf{3}$

The nickel complexes (Scheme 2) were prepared in good yield as shown below in Scheme 2.

General procedure for ethylene polymerization

A 1-1 stainless steel reactor was backed out under $\mathrm{N}_{2}$ flow for at least $1 \mathrm{~h}$ at $150{ }^{\circ} \mathrm{C}$ and subsequently cooled to room temperature for polymerization. After the reactor was

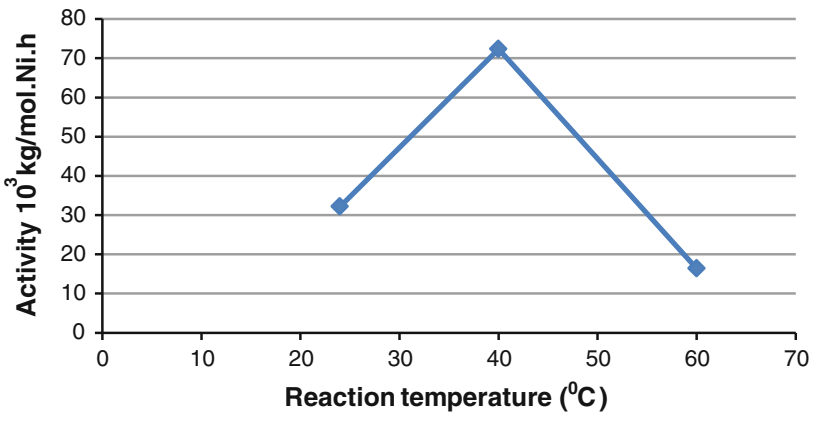

Fig. 1 A plot of reaction temperature versus the catalytic activity of catalyst 2 at ethylene pressure of 10 bars and polymerization time $1 \mathrm{~h}$

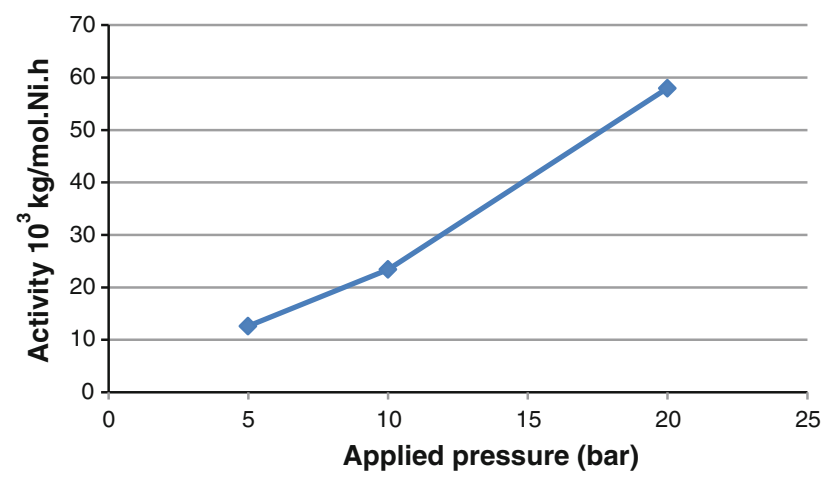

Fig. 2 A plot of applied pressure versus the catalytic activity of catalyst 2 . Polymerization time $1 \mathrm{~h}$ and temperature $24{ }^{\circ} \mathrm{C}$

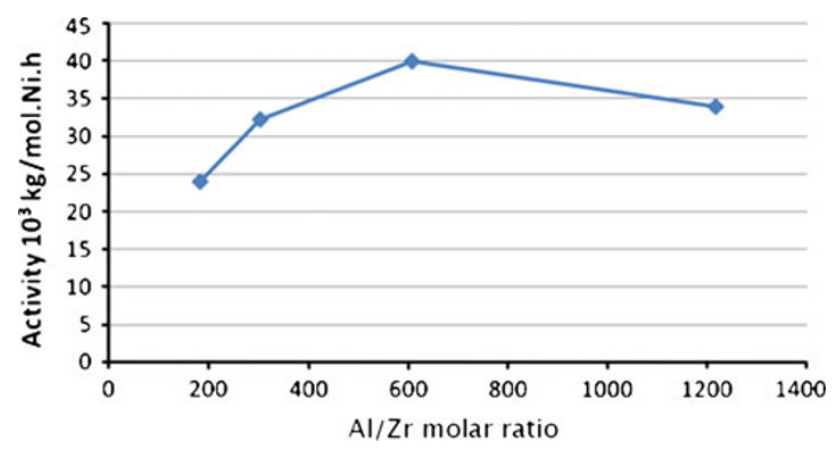

Fig. 3 A plot of Al/Ni ratio versus the catalytic activity of catalyst 2 at ethylene pressure of $10 \mathrm{bars}$, polymerization time $1 \mathrm{~h}$ and temperature $24{ }^{\circ} \mathrm{C}$

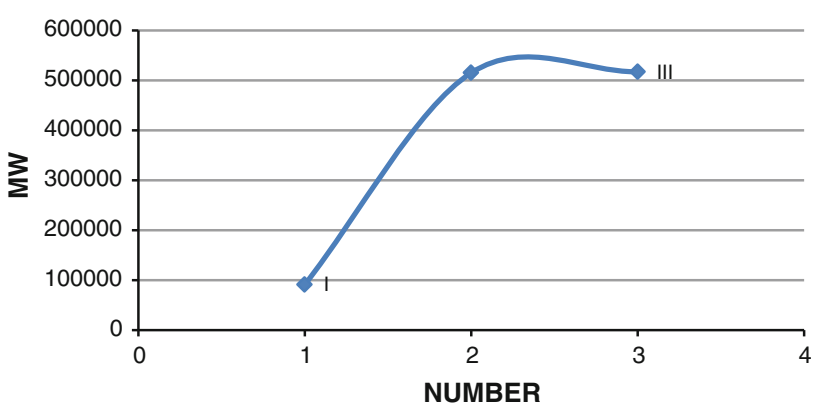

Fig. 4 A plot of molecular weight of polyethylene versus the substituent type

cooled, MAO dissolved in toluene $(50 \mathrm{ml})$ was introduced into the reactor and stirred at reaction temperature. The ethylene was introduced into the reactor by back pressure of nitrogen for at least $1 \mathrm{~h}$. The catalyst solution in toluene (or hexane) was then injected under $\mathrm{N}_{2}$. The reactor pressure was maintained constant throughout the polymerization run by computer-controlled addition of ethylene. The polymerization time was between 20 and $60 \mathrm{~min}$. Runs were terminated by venting off volatiles and the reactor contents were isolated, washed with aqueous $\mathrm{HCl}$ and methanol and the polymer produced was dried in a vacuum oven at $50{ }^{\circ} \mathrm{C}$.

\section{Results and discussion}

Synthesis of new ligands and complexes

The goal of this collaborative research work is to develop and envisage a convenient method to prepare highly active new bi-nuclear alpha-diimine-nickel catalysts toward the catalytic performances for ethylene polymerization. The basic idea is to utilize substituents on the arene moiety and/ or the backbone of the ligand to prepare new ligands and corresponding complexes. The structure around the metal may strongly influence the catalytic performances, such as catalytic activity. Under the guidance of this basic idea, three kinds of new catalysts including bi-nickel-center catalysts, were prepared and studied for catalytic ethylene polymerization in toluene using MAO as co-catalysts.

On treatment with MAO, all the three nickel complexes are active towards ethylene polymerization. They all showed strong temperature dependence of the catalytic activities. Furthermore, they later decrease monotonously with the increase of polymerization temperature (as shown in Figs. 1, 2). In addition, the catalyst's activity increases with the increase of the molar ratio, $\mathrm{Al} / \mathrm{Ni}$, as shown in Fig. 3 where the catalyst activity significantly increased; whereas, when the ratio value increased from 200 to 600 it has little or no effect upon any further increase. The most 
active was the new catalyst with diisopropyl substitute, the catalytic activity was high at $25^{\circ} \mathrm{C}$ with $\mathrm{Al}(\mathrm{MAO}) \mathrm{Ni}$ ratio at 500. In general, the ratio of $\mathrm{Al} / \mathrm{Ni}$ was 500:1 for the most of polymerization reported in this work.

\section{Conclusion}

The result of ethylene polymerization catalyzed by $\mathbf{1 , 2}$ and 3 complexes using MAO as a cocatalyst showed that the activity of complexes $\mathbf{2}$ and $\mathbf{3}$ is higher than that for $\mathbf{1}$ which could be explained by the fact that the ligand environment around the metal center has a profound effect on catalysts' productivity and product molecular weight. Changing the ligand environment results, generally, in a slight drop in productivity and in the molecular weight, as shown by Fig. 4. Confirming the conjugation and steric protection of the metal center are crucial for high activity [1].

Acknowledgments A.A. Alsaygh would like to give thanks and appreciations to King Abdulaziz City for Science and Technology for funding; to his counterparts at Oxford University represented by Peter Edwards and Vladimir Kuznetsov. Moreover, he would like to thank Prof Ibrahim M. Al-Najjar, and Mohammed H. Al-Musawi for their great contributions to this work.

Open Access This article is distributed under the terms of the Creative Commons Attribution License which permits any use, distribution, and reproduction in any medium, provided the original author(s) and the source are credited.

\section{References}

1. Al-Saygh AA, Al-Najjar IM, Al-Musawi MH (2009) 2do SiLQCOM, Maracaibo, Venezuela, from October 25 to 29

2. Britovsek GJP, Bruce M, Gibson VC, Kimberley BS, Maddox PJ, Mastroianni S, McTavish SJ, Redshaw C, Solan GA, Strömberg S, White AJP, Williams DJ (1999) Iron and cobalt ethylene polymerization catalysts bearing 2,6-Bis(imino)pyridyl ligands: synthesis, structures, and polymerization studies. J Am Chem Soc 121:(38)8728-8740

3. Helldorfer M, Backhaus J, Alt HG (2003) New bi-nuclear and multi-nuclear $\alpha$-diimine/nickel catalysts for ethylene polymerization. Inorg Chim Acta 351(2003):34-42

4. Liu J-Y, Zheng Yi, Li Y-G, Pan Li, Li Y-S, Hu N-H (2005) Fe(II) and $\mathrm{Co}(\mathrm{II})$ pyridinebisimine complexes bearing different substituents on ortho- and para-position of imines: synthesis, characterization and behavior of ethylene polymerization. J Organomet Chem 690:1233-1239

5. Luo H-K, Schumann H (2005) Group 4 ansa-cyclopentadienylamido catalysts for olefin polymerization. J Mol Catal A Chem 227:153-161

6. McKnight AL, Waymouth RM (1998) The influence of the ligand structure on the properties of ( $\alpha$-diimine)nickel catalysts in the polymerization and oligomerization of ethylene. Chem Rev 98:2587-2598

7. Gibson VC, Spitzmesser SK (2003) Advances in non-metallocene olefin polymerization catalysis. Chem Rev 103:283-315 